\title{
Integral action for setpoint regulation control of a reaction-diffusion equation in the presence of a state delay $\star$
}

\author{
Hugo Lhachemi ${ }^{\text {a }}$, Ammar Malik ${ }^{\mathrm{b}}$, Robert Shorten ${ }^{\mathrm{b}, \mathrm{c}}$, \\ ${ }^{a}$ Université Paris-Saclay, CNRS, CentraleSupélec, Laboratoire des signaux et systèmes, 91190, Gif-sur-Yvette, France \\ ${ }^{\mathrm{b}}$ School of Electrical and Electronic Engineering, University College Dublin, Dublin, Ireland \\ ${ }^{\mathrm{c}}$ Dyson School of Design Engineering, Imperial College London, London, U.K
}

\begin{abstract}
This paper is concerned with the regulation control of a one-dimensional reaction-diffusion equation in the presence of a state-delay in the reaction term. The objective is to achieve the PI regulation of the right Dirichlet trace with a command selected as the left Dirichlet trace. The control design strategy consists of the design of a PI controller on a finite dimensional truncated model obtained by spectral reduction. By an adequate selection of the number of modes of the original infinitedimensional system, we show that the proposed control design procedure achieves both the exponential stabilization of the original infinite-dimensional system as well as the setpoint regulation of the right Dirichlet trace.
\end{abstract}

Key words: PI regulation; Reaction-diffusion equation; State-delay; Partial differential equation; Input-to-state stability.

\section{Introduction}

The proportional integral (PI) regulation control of infinite-dimensional systems, and in particular of partial differential equations (PDEs), has attracted much attention in the recent years. Early works dealt with bounded control operators [18] while the extension to the case of unbounded control operators was reported in [23]. The last decade has seen an intensification of the efforts in this research direction. PI boundary control of linear hyperbolic systems $[2,6,10,24]$, as well as the extension to nonlinear transport equations [3,22] have been reported. Other types of PDEs have also been studied. This includes reaction-diffusion equations [13], wave equations used to model drilling systems $[1,21]$, as well as semilinear wave equations [14]. The possible addition of an integral action to open-loop stable semigroups was investigated in [20].

\footnotetext{
‡ This publication was supported in part by a research grant from Science Foundation Ireland (SFI) under grant number 16/RC/3872 and is co-funded under the European Regional Development Fund and by I-Form industry partners. The work of the first author was supported by ANR PIA funding: ANR-20-IDEES-0002. Corresponding author H. Lhachemi.

Email addresses: hugo. lhachemi@centralesupelec.fr (Hugo Lhachemi), ammar.malik@ucdconnect.ie (Ammar Malik), r.shorten@imperial.ac.uk (Robert Shorten).
}

We study in this paper the boundary PI regulation control of a reaction-diffusion equation in the presence of a state-delay in the reaction term. Since delays are ubiquitous in practical applications, the topic of boundary stabilization of PDEs in the presence of delays, either in the control input $[9,11,12,17,19]$ or in the state $[7,8,15]$, has also attracted much attention in the recent years. However, it is worth noting that none of the aforementioned works embracing PI control design for PDEs was concerned with the possible presence of state-delays. This paper is a first step in that research direction. Specifically, the objective of this work is to extend the result reported in [15], which solely dealt with the boundary stabilization of a reaction-diffusion equation in the presence of a state-delay, to the PI regulation control of a Dirichlet trace. More precisely we consider the PDE:

$$
\begin{aligned}
& y_{t}(t, x)=a y_{x x}(t, x)+b y(t, x)+c y(t-h(t), x), \\
& y(t, 0)=u(t) \\
& \cos (\theta) y(t, 1)+\sin (\theta) y_{x}(t, 1)=0 \\
& y(\tau, x)=\phi(\tau, x), \quad \tau \in\left[-h_{M}, 0\right]
\end{aligned}
$$

for $t>0$ and $x \in(0,1)$. Here $a>0, b, c \in \mathbb{R}$ with $c \neq 0$, $h: \mathbb{R}_{+} \rightarrow\left[h_{m}, h_{M}\right]$ is continuous with $0<h_{m}<h_{M}$, and $\theta \in(0, \pi / 2)$. The state at time $t$ is $y(t, \cdot):[0,1] \rightarrow \mathbb{R}$. The control input is $u(t) \in \mathbb{R}$ and applies to the left Dirichlet trace (1b). On the right-hand side of the do- 
main, we consider the Robin boundary condition (1c). The initial condition is $\phi:\left[-h_{M}, 0\right] \times(0,1) \rightarrow \mathbb{R}$. The control objective is to design a PI controller in order to stabilize (1) while achieving the setpoint regulation control of the right Dirichlet trace $z(t)=y(t, 1)$. In particular, denoting by $r: \mathbb{R}_{+} \rightarrow \mathbb{R}$ a continuous reference signal, $y(t, 1)$ must achieve the setpoint tracking of $r(t)$.

The strategy for solving the above control design problem goes as follows. Inspired by [4], a finite dimensionaltruncated model is obtained by spectral reduction. The order of the state-delayed truncated model is selected to ensure the stability of the residual infinite-dimensional dynamics. Then, inspired by [13] but with the challenge of a state-delayed term, the truncated model is augmented with an integral component to ensure the setpoint tracking of $z(t)$. Finally, the feedback law is obtained by pole shifting. We assess the exponential stability of the closed-loop system, as well as the setpoint regulation control of the right Dirichlet trace. In the presence of an additive boundary perturbation in the control input, we show that the closed-loop system is exponentially input-to-state stable (ISS). This objective requires to work simultaneously with the original representation of the plant (for ISS purposes w.r.t. boundary disturbances) and an homogeneous version of the PDE (to analyze the system output) while handling the state-delay for both stability and setpoint regulation assessment.

The control design strategy is introduced in Section 2. The equilibrium conditions of the closed-loop system and the related dynamics of deviations are presented in Section 3. The stability analysis is reported in Section 4 while the reference tracking assessment is completed in Section 5. The robustness of the control strategy w.r.t. delay mismatches is studied in Section 6. Finally, numerical simulations are carried out in Section 7 while concluding remarks are formulated in Section 8.

\section{Control design strategy}

\subsection{Spectral reduction and truncated model}

Let $\mathcal{H}=L^{2}(0,1)$ with the inner product $\langle f, g\rangle=$ $\int_{0}^{1} f g \mathrm{~d} x$. System (1) can be rewritten as

$$
\begin{aligned}
& \frac{\mathrm{d} X}{\mathrm{~d} t}(t)=\mathcal{A} X(t)+c X(t-h(t)), \\
& \mathcal{B} X(t)=u(t), \\
& X(\tau)=\Phi(\tau)=\phi(\tau, \cdot), \quad \tau \in\left[-h_{M}, 0\right]
\end{aligned}
$$

for $t \geq 0$ with $\mathcal{A}: D(\mathcal{A}) \subset \mathcal{H} \rightarrow \mathcal{H}$ defined on $D(\mathcal{A})=$ $\left\{f \in H^{2}(0,1): \cos (\theta) f(1)+\sin (\theta) f^{\prime}(1)=0\right\}$, with $\theta \in$ $(0, \pi / 2)$, by $\mathcal{A} f=a f^{\prime \prime}+b f$ and the boundary operator $\mathcal{B}: D(\mathcal{B}) \subset \mathcal{H} \rightarrow \mathbb{R}$ defined on $D(\mathcal{B})=H^{1}(0,1)$ by $\mathcal{B} f=f(0)$. We define the disturbance free operator $\mathcal{A}_{0}=\left.\mathcal{A}\right|_{D\left(\mathcal{A}_{0}\right)}$ on $D\left(\mathcal{A}_{0}\right)=D(\mathcal{A}) \cap \operatorname{ker}(\mathcal{B})$. It is well-known that $\mathcal{A}_{0}$ generates a $C_{0}$-semigroup. We introduce $L \in \mathcal{L}(\mathbb{R}, \mathcal{H})$ defined for any $u \in \mathbb{R}$ by $[L u](x)=$ $(1-x)^{2} u, x \in[0,1]$. $L$ has been selected such that its range satisfies $\mathrm{R}(L) \subset D(\mathcal{A})$ and $\mathcal{B} L=I_{\mathbb{R}}$. Hence, following the terminology of $[5$, Sec. 3.3], the pair $(\mathcal{A}, \mathcal{B})$ defines a boundary control system with associated lifting operator $L$. We define $\mathcal{A}_{c} \triangleq \mathcal{A}+c I_{\mathcal{H}}$ and $\mathcal{A}_{c, 0} \triangleq \mathcal{A}_{0}+c I_{\mathcal{H}}$ on $D\left(\mathcal{A}_{c}\right)=D(\mathcal{A})$ and $D\left(\mathcal{A}_{c, 0}\right)=D\left(\mathcal{A}_{0}\right)$, respectively. From the Sturm-Liouville theory, it well known that the eigenvalues of $\mathcal{A}_{c, 0}$ are simple and form a decreasing sequence $\left(\lambda_{n}\right)_{n \geq 0} \in \mathbb{R}^{\mathbb{N}}$ with $\lambda_{n} \rightarrow-\infty$ when $n \rightarrow+\infty$. Moreover, one can select the associated eigenvectors such that $\left(e_{n}\right)_{n>0}$ forms a Hilbert basis of $\mathcal{H}$. Using the terminology of $\left[\overline{5}\right.$, Def. 2.3.4], $\mathcal{A}_{c, 0}$ is a Riesz spectral operator: $D\left(\mathcal{A}_{c, 0}\right)=\left\{f \in L^{2}(0,1): \sum_{n \geq 0}\left|\lambda_{n}\right|^{2}\left|\left\langle f, e_{n}\right\rangle\right|^{2}<\infty\right\}$ and $\mathcal{A}_{c, 0} f=\sum_{n>0} \lambda_{n}\left\langle f, e_{n}\right\rangle e_{n}$ for all $f \in D\left(\mathcal{A}_{c, 0}\right)$. Standard computations give $\lambda_{n}=b+c-a r_{n}^{2}$ and $e_{n}=$ $2 \sqrt{\frac{r_{n}}{2 r_{n}-\sin \left(2 r_{n}\right)}} \sin \left(r_{n} \cdot\right)$ with $n \in \mathbb{N}$ where $r_{n}>0$ is the unique number $r \in(n \pi,(n+1) \pi)$ such that $r \cot (r)=$ $-\cot (\theta)$. This yields $\lambda_{n} \sim-a n^{2} \pi^{2}$ and $e_{n}(1)=O(1)$ as $n \rightarrow+\infty$. Introducing $x_{n}(t)=\left\langle X(t), e_{n}\right\rangle$ the coefficients of projection of the system trajectory into the Hilbert basis, we have that $X(t)=\sum_{n>0} x_{n}(t) e_{n}$ and $\|X(t)\|^{2}=\sum_{n>0}\left|x_{n}(t)\right|^{2}$. Assuming that $u$ is continuously differentiable and $\Phi$ is continuous, the mild solution $X \in \mathcal{C}^{0}\left(\mathbb{R}_{+} ; \mathcal{H}\right)$ of $(2)$ is such that $x_{n}$ is continuously differentiable and (see [15] for details)

$\dot{x}_{n}(t)=\lambda_{n} x_{n}(t)+c\left\{x_{n}(t-h(t))-x_{n}(t)\right\}+\left(a_{n}+\lambda_{n} b_{n}\right) u(t)$

with

$$
a_{n}=\left\langle\mathcal{A}_{c} L \mathbb{1}, e_{n}\right\rangle, \quad b_{n}=-\left\langle L \mathbb{1}, e_{n}\right\rangle
$$

where $\mathbb{1}$ denotes here the unit element of $\mathbb{R}$. Note that due to the presence of the state-delay, there may exist delays for which certain modes $x_{n}$, hence the PDE, are unstable even if $\lambda_{n}-c=b-a r_{n}^{2}<0$ and $c<0$ provided $c$ is large enough [16, Sec. 3.3]. For a given integer $N \geq 0$ selected such that $\lambda_{n}<0$ for all $n \geq N+1$ and which will be further constrained later, we define the followings:

$$
\begin{aligned}
Y(t) & =\left[x_{0}(t) \ldots x_{N}(t)\right]^{\top} \in \mathbb{R}^{N+1}, \\
Y_{\Phi}(\tau) & =\left[\left\langle\Phi(\tau), e_{0}\right\rangle \ldots\left\langle\Phi(\tau), e_{N}\right\rangle\right]^{\top} \in \mathbb{R}^{N+1}, \\
A & =\operatorname{diag}\left(\lambda_{n}\right)_{0 \leq n \leq N} \in \mathbb{R}^{(N+1) \times(N+1)}, \\
B & =\left(a_{n}+\lambda_{n} b_{n}\right)_{0 \leq n \leq N} \in \mathbb{R}^{(N+1)} .
\end{aligned}
$$

Then we obtain the truncated model:

$$
\begin{aligned}
\dot{Y}(t) & =A Y(t)+c\{Y(t-h(t))-Y(t)\}+B u(t) \\
Y(\tau) & =Y_{\Phi}(\tau), \quad \tau \in\left[-h_{M}, 0\right]
\end{aligned}
$$

1 Such a regularity for the forthcoming control law will be assessed in the sequel. 


\subsection{Addition of an integral component}

The objective is now to augment the truncated model (6) with an integral component to achieve the setpoint regulation control of the right Dirichlet trace $z(t)=y(t, 1)$.

We first need to express the right Dirichlet trace $y(t, 1)$ in function of the coefficients of projection $x_{n}$.

Lemma 1 Let $\theta \in(0, \pi / 2)$. For all $f \in D\left(\mathcal{A}_{c, 0}\right)$ we have $f(1)=\sum_{n \geq 0}\left\langle f, e_{n}\right\rangle e_{n}(1)$.

The proof of this Lemma, which is omitted, essentially relies on the Riesz-spectral property of $\mathcal{A}_{c, 0}$. We cannot directly apply the above series expansion to the trajectory $X$ of our system because, in general, $X(t) \notin D\left(\mathcal{A}_{c, 0}\right)$. However, if we assume that $X \in \mathcal{C}^{0}\left(\mathbb{R}_{+} ; D(\mathcal{A})\right) \cap \mathcal{C}^{1}\left(\mathbb{R}_{+} ; \mathcal{H}\right)$ is a classical solution of $(2)$, one has $W(t)=X(t)-L u(t) \in D\left(\mathcal{A}_{0}\right)=D\left(\mathcal{A}_{c, 0}\right)$ with in particular $y(t, 1)=[X(t)](1)=[W(t)](1)$. Hence, introducing $w_{n}(t)=\left\langle W(t), e_{n}\right\rangle=x_{n}(t)+b_{n} u(t)$, we obtain that $y(t, 1)=[X(t)](1)=\sum_{n \geq 0} w_{n}(t) e_{n}(1)$ for all $t \geq 0$. Since $u$ is of class $\mathcal{C}^{1}$ (we will actually need $u$ of class $\mathcal{C}^{2}$ to ensure the existence of classical solutions), we have that $w_{n}$ is of class $\mathcal{C}^{1}$ and, from (3),

$$
\begin{aligned}
\dot{w}_{n}(t)= & \lambda_{n} w_{n}(t)+c\left\{w_{n}(t-h(t))-w_{n}(t)\right\}+a_{n} u(t) \\
& -c b_{n}\{u(t-h(t))-u(t)\}+b_{n} \dot{u}(t)
\end{aligned}
$$

for $t \geq h_{M}$.

Remind that our objective is to achieve the setpoint regulation control of the system output $z(t)=y(t, 1)$. In order to introduce in a comprehensive manner the proposed integral component $\zeta(t) \in \mathbb{R}$ that will be used to augment the truncated model $(6)$, consider first the classical integral component given by $\dot{\chi}(t)=y(t, 1)-$ $r(t)=\sum_{n \geq 0} w_{n}(t) e_{n}(1)-r(t)$ for $t \geq 0$. Here $r(t) \in \mathbb{R}$ stands for a reference signal. Recall that the second equality holds only when considering classical solutions for (2). As the above series expansion involves all the modes of the system, and in particular the coefficients of projection $w_{n}(t)$ for $n \geq N+1$, the integral component $\chi$ cannot be directly included into the dynamics of the truncated model (6). To solve this issue, we introduce the following preliminary change of variable $\zeta_{p}(t)=\chi(t)+\sum_{n \geq N+1} \frac{e_{n}(1)}{\lambda_{n}}\left\{b_{n} u(t)-w_{n}(t)\right\}$. Note that the convergence of the series follow from $\lambda_{n} \sim$ $-a n^{2} \pi^{2}$ and $e_{n}(1)=O(1)$ as $n \rightarrow+\infty$. Based on (7) we obtain, for $t \geq h_{M}, \dot{\zeta}_{p}(t)=\sum_{n=0}^{N} x_{n}(t) e_{n}(1)+$ $\alpha u(t)-r(t)-c \sum_{n \geq N+1} \frac{e_{n}(1)}{\lambda_{n}}\left\{w_{n}(t-h(t))-w_{n}(t)\right\}+$ $c \sum_{n \geq N+1} \frac{e_{n}(1)}{\lambda_{n}} b_{n}\{u(t-h(t))-u(t)\}$ where

$$
\alpha=\sum_{n=0}^{N} b_{n} e_{n}(1)-\sum_{n \geq N+1} \frac{a_{n}}{\lambda_{n}} e_{n}(1) .
$$

We now note that the two last terms of the above identity describing the $\zeta_{p}$-dynamics have a null contribution at equilibrium. This observation motivates the introduction of the below $\zeta$-dynamics. Assuming that the delay $h$ is known (robustness w.r.t. delay mismatches will be discussed later in Section 6), we mimic the structure of the dynamics of the truncated model (6) by defining for $t \geq 0$ the integral component $\zeta(t) \in \mathbb{R}$ as follows:

$$
\begin{aligned}
\dot{\zeta}(t)= & \sum_{n=0}^{N} x_{n}(t) e_{n}(1)+c\{\zeta(t-h(t))-\zeta(t)\} \\
& +\alpha u(t)-r(t), \\
\zeta(\tau)= & \zeta_{0}(\tau), \quad \tau \in\left[-h_{M}, 0\right]
\end{aligned}
$$

Remark 1 The $\zeta$-dynamics achieves the same equilibrium condition as the $\zeta_{p}$-dynamics. As we will show later in Section 3, the integral component (9) ensures that the equilibirum condition $\left(X_{e}, \zeta_{e}\right)$ of the forthcoming closedloop system, associated with some constant reference signal $r(t)=r_{e}$, achieves the desired reference tracking for the right Dirichlet trace, i.e., $X_{e}(1)=r_{e}$.

Remark 2 Even if (9) has been motivated and derived by considering classical solutions of (2), the dynamics (9) actually makes sense for any mild solutions of (2).

Since (9) only involves the $N+1$ first modes of the system, we can now augment the dynamics of the truncated model (6) with the $\zeta$-dynamics as follows:

$$
\begin{aligned}
\dot{Y}_{a}(t)= & A_{a} Y_{a}(t)+c\left\{Y_{a}(t-h(t))-Y_{a}(t)\right\} \\
& +B_{a} u(t)+\Gamma(t), \\
Y_{a}(\tau)= & Y_{\Phi, a}(\tau), \quad \tau \in\left[-h_{M}, 0\right]
\end{aligned}
$$

where $C=\left[e_{0}(1) \ldots e_{N}(1)\right] \in \mathbb{R}^{1 \times(N+1)}$,

$$
\begin{gathered}
Y_{a}(t)=\left[\begin{array}{c}
Y(t) \\
\zeta(t)
\end{array}\right], \quad Y_{\Phi, a}(\tau)=\left[\begin{array}{c}
Y_{\Phi}(\tau) \\
\zeta_{0}(\tau)
\end{array}\right], \\
A_{a}=\left[\begin{array}{ll}
A & 0 \\
C & 0
\end{array}\right], \quad B_{a}=\left[\begin{array}{l}
B \\
\alpha
\end{array}\right], \quad \Gamma(t)=\left[\begin{array}{c}
0 \\
-r(t)
\end{array}\right] .
\end{gathered}
$$

\subsection{Proposed control strategy}

The proposed control strategy consists of a stabilizing state feedback of the truncated model (10). Such a procedure is allowed by the following lemma.

Lemma $2\left(A_{a}, B_{a}\right)$ satisfies the Kalman condition.

Proof. We define the matrix $T=\left[\begin{array}{ll}A & B \\ C & \alpha\end{array}\right] \in \mathbb{R}^{(N+2) \times(N+2)}$.

From (11), the Hautus test shows that the pair 
$\left(A_{a}, B_{a}\right)$ satisfies the Kalman condition if an only if $T$ is invertible and the pair $(A, B)$ satisfies the Kalman condition. To show the former, let $Y_{*}=$ $\left[\begin{array}{llll}x_{*, n} & \ldots & x_{*, N} & u_{*}\end{array}\right]^{\top} \in \operatorname{ker}(T)$. From (5) and (11) we deduce that $\lambda_{n} x_{*, n}+\left(a_{n}+\lambda_{n} b_{n}\right) u_{*}=0$ for all $0 \leq n \leq N$ and $\sum_{n=0}^{N} x_{*, n} e_{n}(1)+\alpha u_{*}=0$. Since $\lambda_{n}<0$ for all $n \geq N+1$, we define $x_{*, n}=-\frac{a_{n}+\lambda_{n} b_{n}}{\lambda_{n}} u_{*}$ for all $n \geq N+1$. Hence we have $\lambda_{n} x_{*, n}+\left(a_{n}+\lambda_{n} b_{n}\right) u_{*}=0$ for all $n \geq 0$. We also define $w_{*, n}=x_{*, n}+b_{n} u_{*}$ that gives $\lambda_{n} w_{*, n}+a_{n} u_{*}=0$ for all $n \geq 0$. We infer that $\left(w_{*, n}\right)_{n}$ and $\left(\lambda_{n} w_{*, n}\right)_{n}$ are in $\ell^{2}(\mathbb{N})$, hence we can define $w_{*}=\sum_{n>0} w_{*, n} e_{n} \in D\left(\mathcal{A}_{c, 0}\right)$. Moreover, the latter equation shows that $\mathcal{A}_{c, 0} w_{*}+\mathcal{A}_{c} L u_{*}=0$. Using now the definition of $\alpha$ given by (8), we deduce that $0=\sum_{n=0}^{N} x_{*, n} e_{n}(1)+\alpha u_{*}=\sum_{n \geq 0} w_{*, n} e_{n}(1)=w_{*}(1)$. Therefore, introducing $x_{*}=w_{*}+L u_{*} \in D\left(\mathcal{A}_{c}\right)$, we obtain that $\mathcal{A}_{c} x_{*}=0$ and $x_{*}(1)=w_{*}(1)+\left[L u_{*}\right](1)=0$. This shows that $a x_{*}^{\prime \prime}+(b+c) x_{*}=0$ with $x_{*}(1)=0$ and $x_{*}^{\prime}(1)=-\cot (\theta) x_{*}(1)=0$. So, by Cauchy uniqueness, $x_{*}=0$. Since $w_{*}(0)=0$, we get $0=x_{*}(0)=\left[L u_{*}\right](0)=$ $u_{*}$. We infer $w_{*}=0$ hence $w_{*, n}=0$ for all $n \geq 0$. This implies that $x_{*, n}=w_{*, n}-b_{n} u_{*}=0$ for all $n \geq 0$. We deduce that $Y_{*}=0$, which shows that $T$ in invertible.

We now show that $(A, B)$ satisfies the Kalman condition. In view of (5), since $A$ is diagonal with simple eigenvalues, it is sufficient to show that $a_{n}+\lambda_{n} b_{n} \neq 0$ for all $n \geq 0$. From (4), using two integration by parts and the identity $\mathcal{A}_{c, 0} e_{n}=\lambda_{n} e_{n}$, we obtain that $a_{n}+\lambda_{n} b_{n}=$ $a e_{n}^{\prime}(0)$. Since $e_{n} \neq 0$ with $e_{n}(0)=0$, we obtain by Cauchy uniqueness that $e_{n}^{\prime}(0) \neq 0$ hence $a_{n}+\lambda_{n} b_{n} \neq 0$ for all $n \geq 0$. Thus $(A, B)$ satisfies the Kalman condition, which completes the proof.

Thus there exists $K \in \mathbb{R}^{1 \times(N+2)}$ such that $A_{K}=A_{a}+$ $B_{a} K$ is Hurwitz with simple eigenvalues. We set for $t \geq 0$

$$
u(t)=K Y_{a}(t)+p(t)
$$

where $p$ is a boundary disturbance. The control (12) takes the form of a PI controller because composed of 1) a proportional feedback of the state, via $Y(t)$, and 2$)$ the integral component $\zeta(t)$ given by $(9)$. We now need to select the integer $N \geq 0$ such that the closed-loop system composed of (2), (9), and (12), is exponentially input-to-state stable with respect to the boundary perturbation $p$ and achieves the setpoint reference tracking of the system output $z(t)=y(t, 1)$.

\subsection{Well-posedness of the closed-loop system dynamics}

The study of the well-posedness of the closed-loop system, which requires the introducing of the augmented state $X_{\zeta}=(X, \zeta)$ belonging to $\mathcal{H}_{\zeta}=L^{2}(0,1) \times \mathbb{R}$ endowed with the inner product $\left\langle\left(f, \zeta_{f}\right),\left(g, \zeta_{g}\right)\right\rangle_{\zeta}=$ $\int_{0}^{1} f g \mathrm{~d} x+\zeta_{f} \zeta_{g}$, easily leads to the following result.
Lemma 3 Let $0<h_{m}<h_{M}, h \in \mathcal{C}^{0}\left(\mathbb{R}_{+}\right)$with $h_{m} \leq$ $h(t) \leq h_{M}, \Phi \in \mathcal{C}^{0}\left(\left[-h_{M}, 0\right] ; \mathcal{H}\right), \zeta_{0} \in \mathcal{C}^{0}\left(\left[-h_{M}, 0\right]\right)$, $p \in \mathcal{C}^{1}\left(\mathbb{R}_{+}\right)$, and $r \in \mathcal{C}^{0}\left(\mathbb{R}_{+}\right)$. Then there exists a unique mild solution $X_{\zeta}=(X, \zeta) \in \mathcal{C}^{0}\left(\mathbb{R}_{+} ; \mathcal{H}_{\zeta}\right)$ of (2) and (9) with control input (12). Moreover we have $u, \zeta \in \mathcal{C}^{1}\left(\mathbb{R}_{+}\right)$.

To assess the setpoint regulation, we need to resort to the concept of classical solutions. The existence and uniqueness of such solutions is guaranteed by the following corollary whose proof is an immediate consequence of classical results, see, e.g., [5, Thm. 3.1.3].

Corollary 1 Let $0<h_{m}<h_{M}, h \in \mathcal{C}^{1}\left(\mathbb{R}_{+}\right)$with $h_{m} \leq h(t) \leq h_{M}$ and such that $t \mapsto t-h(t)$ crosses 0 a finite number of times, $\Phi \in \mathcal{C}^{1}\left(\left[-h_{M}, 0\right] ; \mathcal{H}\right), \zeta_{0} \in$ $\mathcal{C}^{1}\left(\left[-h_{M}, 0\right]\right), p \in \mathcal{C}^{2}\left(\mathbb{R}_{+}\right)$, and $r \in \mathcal{C}^{1}\left(\mathbb{R}_{+}\right)$. Assume that $\Phi(0) \in D(\mathcal{A})$ so that the compatibility condition

$$
\mathcal{B} \Phi(0)=K Y_{\Phi, a}(0)+p(0)
$$

holds. Then there exists a unique classical solution $X_{\zeta}=$ $(X, \zeta) \in \mathcal{C}^{0}\left(\mathbb{R}_{+} ; D(\mathcal{A}) \times \mathbb{R}\right) \cap \mathcal{C}^{1}\left(\mathbb{R}_{+} ; \mathcal{H}_{\zeta}\right)$ of (2) and (9) with control input (12). Moreover we have $u \in \mathcal{C}_{\mathrm{pw}}^{2}\left(\mathbb{R}_{+}\right)$.

Remark 3 From (11) and because $A_{K}=A_{a}+B_{a} K$ is Hurwitz hence invertible, the last coefficient of $K$, that corresponds to the integral state $\zeta$, is necessarily non zero. Hence, for any given initial condition $\Phi \in$ $\mathcal{C}^{1}\left(\left[-h_{M}, 0\right] ; \mathcal{H}\right)$ with $\Phi(0) \in D(\mathcal{A})$ and any boundary perturbation $p \in \mathcal{C}^{2}\left(\mathbb{R}_{+}\right)$, one can always select the initial condition $\zeta_{0} \in \mathcal{C}^{1}\left(\left[-h_{M}, 0\right]\right)$ of the integral component such that the compatibility condition (13) holds.

\section{Equilibrium conditions and associated dy- namics of deviations}

\subsection{Equilibrium conditions}

Let $r_{e}, p_{e} \in \mathbb{R}$ be "nominal" values of the reference signal $r(t)$ and the boundary perturbation $p(t)$, respectively. Our first objective is to derive the equilibrium condition of the closed-loop system when setting $r(t)=r_{e}$ and $p(t)=p_{e}$. To do so, we denote by the subscript "e" the equilibrium condition associated with the different system signals. We define $Y_{a, e}=\left[Y_{e}^{\top} \zeta_{e}\right]^{\top}, Y_{e}=$ $\left[x_{0, e} \ldots x_{N, e}\right]^{\top}$, and $\Gamma_{e}=\left[0-r_{e}\right]^{\top}$. From (10) and (12) we set $Y_{a, e}=-A_{K}^{-1}\left(B_{a} p_{e}+\Gamma_{e}\right)$ and $u_{e}=K Y_{a, e}+p_{e}$ which give $0=A_{a} Y_{a, e}+B_{a} u_{e}+\Gamma_{e}$. From (5) and (11), this implies that $0=\lambda_{n} x_{n, e}+\left(a_{n}+\lambda_{n} b_{n}\right) u_{e}$ for all $0 \leq$ $n \leq N$ and $0=\sum_{n=0}^{N} x_{n, e} e_{n}(1)+\alpha u_{e}-r_{e}$. Regarding the residual dynamics given by (3) for $n \geq N+1$, we define $x_{n, e}=-\frac{a_{n}+\lambda_{n} b_{n}}{\lambda_{n}} u_{e}$. This yields $0=\lambda_{n} x_{n, e}+\left(a_{n}+\right.$ $\left.\lambda_{n} b_{n}\right) u_{e}$ for all $n \geq 0$. We note that $\left(x_{n, e}\right)_{n \geq 0} \in \ell^{2}(\mathbb{N})$ hence we can define $X_{e}=\sum_{n \geq 0} x_{n, e} e_{n} \in \mathcal{H}$. Moreover, 
introducing for $n \geq 0$ the quantities $w_{n, e}=x_{n, e}+b_{n} u_{e}$, we have for $n \geq N+1$ that $w_{n, e}=-\frac{a_{n}}{\lambda_{n}} u_{e}$, showing that $\left(w_{n, e}\right)_{n \geq 0} \in \ell^{2}(\mathbb{N})$ and $\left(\lambda_{n} w_{n, e}\right)_{n \geq 0} \in \ell^{2}(\mathbb{N})$. This allows the introduction of $W_{e}=\sum_{n \geq 0} w_{n, e} e_{n} \in$ $D\left(\mathcal{A}_{0}\right)=D\left(\mathcal{A}_{c, 0}\right)$. Moreover, from the definition of $b_{n}$ given by (4), we have $X_{e}=W_{e}+L u_{e} \in D\left(\mathcal{A}_{c}\right)$ hence $\mathcal{B} X_{e}=u_{e}$. Furthermore, since $\lambda_{n} w_{n, e}+a_{n} u_{e}=0$ for all $n \geq 0$, we have from the definition of $a_{n}$ given by (4) that $\overline{\mathcal{A}}_{c, 0} W_{e}+\mathcal{A}_{c} L u_{e}=0$ hence $\mathcal{A}_{c} X_{e}=0$. Using now Lemma 1, (8), and the above relations between $x_{n, e}$ and $w_{n, e}$, we obtain from $0=\sum_{n=0}^{N} x_{n, e} e_{n}(1)+\alpha u_{e}-r_{e}$ that $W_{e}(1)=r_{e}$. Since $X_{e} \in D\left(\mathcal{A}_{c}\right) \subset H^{1}(0,1)$, we infer that $X_{e}(1)=W_{e}(1)+\left[L u_{e}\right](1)=r_{e}$, which provides the desired reference tracking.

\subsection{Dynamics of deviations}

Let $r_{e}, p_{e} \in \mathbb{R}$ be arbitrary and consider the different equilibrium quantities defined above. We can introduce the dynamics of deviations of the system trajectory with respect to the considered equilibrium condition. These deviations are denoted by the symbol " $\Delta$ ". For instance, $\Delta X(t)$ stands for $X(t)-X_{e}$. We obtain the following dynamics of deviation: $\frac{\mathrm{d}(\Delta X)}{\mathrm{d} t}(t)=\mathcal{A} \Delta X(t)+c \Delta X(t-$ $h(t)), \mathcal{B} \Delta X(t)=\Delta u(t), \Delta \dot{\zeta}(t)=\sum_{n=0}^{N} \Delta x_{n}(t) e_{n}(1)+$ $c\{\Delta \zeta(t-h(t))-\Delta \zeta(t)\}+\alpha \Delta u(t)-\Delta r(t), \Delta x_{n}(t)=$ $\left\langle\Delta X(t), e_{n}\right\rangle$ and $\Delta w_{n}(t)=\left\langle\Delta W(t), e_{n}\right\rangle=\Delta x_{n}(t)+$ $b_{n} \Delta u(t)$. This yields the following representation for the closed-loop system dynamics:

$$
\begin{aligned}
\Delta \dot{Y}_{a}(t)= & A_{K} \Delta Y_{a}(t)+c\left\{\Delta Y_{a}(t-h(t))-\Delta Y_{a}(t)\right\} \\
& +B_{a} \Delta p(t)+\Delta \Gamma(t), \\
\Delta \dot{x}_{n}(t)= & \lambda_{n} \Delta x_{n}(t)+c\left\{\Delta x_{n}(t-h(t))-\Delta x_{n}(t)\right\} \\
& +\left(a_{n}+\lambda_{n} b_{n}\right) \Delta u(t), \quad n \geq N+1, \quad(1) \\
\Delta u(t)= & K \Delta Y_{a}(t)+\Delta p(t) \\
\Delta Y_{a}(\tau)= & \Delta Y_{\Phi, a}(\tau), \quad \tau \in\left[-h_{M}, 0\right] \\
\Delta x_{n}(\tau)= & \left\langle\Delta \Phi(\tau), e_{n}\right\rangle, \quad \tau \in\left[-h_{M}, 0\right], \quad n \geq 0
\end{aligned}
$$

\section{Stability analysis}

The main result of this section is stated as follows.

Theorem 1 Let $0<h_{m}<h_{M}$ be arbitrarily given. Let $N \geq 0$ be such that $\lambda_{N+1}<-2 \sqrt{5}|c|$ and consider the matrices $A_{a}$ and $B_{a}$ defined by (11). Let $K \in \mathbb{R}^{1 \times(N+2)}$ be such that $A_{K}=A_{a}+B_{a} K$ is Hurwitz with simple eigenvalues $\mu_{1}, \ldots, \mu_{N+2} \in \mathbb{C}$ satisfying $\operatorname{Re} \mu_{n}<-3|c|$ for all $1 \leq n \leq N+2$. Then, there exist constants $\kappa, \bar{C}_{0}, \bar{C}_{1}>0$ such that, for all $h \in \mathcal{C}^{0}\left(\mathbb{R}_{+}\right)$with $h_{m} \leq h(t) \leq h_{M}, \Phi \in$ $\mathcal{C}^{0}\left(\left[-h_{M}, 0\right] ; \mathcal{H}\right), \zeta_{0} \in \mathcal{C}^{0}\left(\left[-h_{M}, 0\right]\right), p \in \mathcal{C}^{1}\left(\mathbb{R}_{+}\right)$, and $r \in \mathcal{C}^{0}\left(\mathbb{R}_{+}\right)$, the mild solution $X_{\zeta}=(X, \zeta) \in \mathcal{C}^{0}\left(\mathbb{R}_{+} ; \mathcal{H}_{\zeta}\right)$ of (2) and (9) with control input (12) satisfies, for all $t \geq 0$

$$
\| \Delta X(t)||+|\Delta \zeta(t)|+|\Delta u(t)|
$$

$$
\begin{aligned}
\leq & \bar{C}_{0} e^{-\kappa t} \sup _{\tau \in\left[-h_{M}, 0\right]}\left(\|\Delta \Phi(\tau)\|+\left|\Delta \zeta_{0}(\tau)\right|\right) \\
& +\bar{C}_{1} \sup _{\tau \in[0, t]} e^{-\kappa(t-\tau)}(|\Delta p(\tau)|+|\Delta r(\tau)|) .
\end{aligned}
$$

Corollary 2 In the context of Theorem 1, assume that $r(t) \rightarrow r_{e}$ and $p(t) \rightarrow p_{e}$ as $t \rightarrow+\infty$. Then $X(t) \rightarrow X_{e}$ and $\zeta(t) \rightarrow \zeta_{e}$ as $t \rightarrow+\infty$ with exponential vanishing of the contribution of the initial conditions.

Remark 4 From Theorem 1, one needs to start by selecting the integer $N \geq 0$ such that $\lambda_{N+1}<-2 \sqrt{5}|c|$. This is always possible because $\lambda_{n} \sim-a n^{2} \pi^{2}$ as $n \rightarrow+\infty$ with $a>0$. Then, because of Lemma 2, the feedback gain $K \in \mathbb{R}^{1 \times(N+2)}$ can always be computed such that $A_{K}=A_{a}+B_{a} K$ is Hurwitz with arbitrary eigenvalue assignment. This allows the application of Theorem 1.

\subsection{Truncated model}

The design of the feedback gain $K$ and the resulting stability properties of the truncated model (14a) rely on the following lemma whose proof is identical to $[15$, Lem. 8].

Lemma 4 Let $N \geq 1,0<h_{m}<h_{M}, \mathbf{A} \in \mathbb{R}^{N \times N}$, and $c \in \mathbb{R}$. Assume that $\mathbf{A}$ is Hurwitz with simple eigenvalues $\mu_{1}, \ldots, \mu_{N} \in \mathbb{C}$ such that $\operatorname{Re} \mu_{n}<-3|c|$ for all $1 \leq$ $n \leq N$. Then there exist $\sigma, C_{0}, C_{1}>0$ such that, for any $x_{0} \in \mathcal{C}^{0}\left(\left[-h_{M}, 0\right] ; \mathbb{R}^{N}\right)$, any $h \in \mathcal{C}^{0}\left(\mathbb{R}_{+}\right)$with $h_{m} \leq$ $h(t) \leq h_{M}$, and any $q \in \mathcal{C}^{0}\left(\mathbb{R}_{+} ; \mathbb{R}^{N}\right)$, the trajectory of

$$
\begin{aligned}
\dot{x}(t) & =\mathbf{A} x(t)+c\{x(t-h(t))-x(t)\}+q(t), \\
x(\tau) & =x_{0}(\tau), \quad \tau \in\left[-h_{M}, 0\right]
\end{aligned}
$$

satisfies, for all $t \geq 0$,

$$
\begin{aligned}
\|x(t)\| \leq & C_{0} e^{-\sigma t} \sup _{\tau \in\left[-h_{M}, 0\right]}\left\|x_{0}(\tau)\right\| \\
& +C_{1} \sup _{\tau \in[0, t]} e^{-\sigma(t-\tau)}\|q(\tau)\| .
\end{aligned}
$$

From the assumptions of Thm. 1, Lemma 4 applies to the truncated model (14a) with initial condition (14d).

\subsection{Residual infinite-dimensional dynamics}

We now need to investigate the selection of the integer $N \geq 0$ such that the residual dynamics composed of $(14 \mathrm{~b})$ and (14e) is exponentially stable.

Lemma 5 Let $0<h_{m}<h_{M}$ and $\sigma, C_{2}, C_{3}>0$ be arbitrarily given. Let $N \geq 0$ be such that $\lambda_{N+1}<-2 \sqrt{5}|c|$. Then, there exist constants $\kappa \in(0, \sigma)$ and $C_{4}, C_{5}>0$ such that, for all $h \in \mathcal{C}^{0}\left(\mathbb{R}_{+}\right)$with $h_{m} \leq h(t) \leq h_{M}$, 
$\Phi \in \mathcal{C}^{0}\left(\left[-h_{M}, 0\right] ; \mathcal{H}\right), \zeta_{0} \in \mathcal{C}^{0}\left(\left[-h_{M}, 0\right]\right), p \in \mathcal{C}^{1}\left(\mathbb{R}_{+}\right)$, $r \in \mathcal{C}^{0}\left(\mathbb{R}_{+}\right)$, and $u \in \mathcal{C}^{1}\left(\mathbb{R}_{+}\right)$such that

$$
\begin{aligned}
|\Delta u(t)| \leq & C_{2} e^{-\sigma t} \sup _{\tau \in\left[-h_{M}, 0\right]}\left(\|\Delta \Phi(\tau)\|+\left|\Delta \zeta_{0}(\tau)\right|\right) \\
& +C_{3} \sup _{\tau \in[0, t]} e^{-\sigma(t-\tau)}(|\Delta p(\tau)|+|\Delta r(\tau)|)
\end{aligned}
$$

for all $t \geq 0$, the mild solution $X_{\zeta}=(X, \zeta) \in \mathcal{C}^{0}\left(\mathbb{R}_{+} ; \mathcal{H}_{\zeta}\right)$ of (2) and (9) satisfies for all $t \geq 0$

$$
\begin{aligned}
& \sum_{n \geq N+1}\left|\Delta x_{n}(t)\right|^{2} \\
& \leq C_{4} e^{-2 \kappa t} \sup _{\tau \in\left[-h_{M}, 0\right]}\left(\|\Delta \Phi(\tau)\|+\left|\Delta \zeta_{0}(\tau)\right|\right)^{2} \\
& \quad+C_{5} \sup _{\tau \in[0, t]} e^{-2 \kappa(t-\tau)}(|\Delta p(\tau)|+|\Delta r(\tau)|)^{2} .
\end{aligned}
$$

Remark 5 The design constraint $\lambda_{N+1}<-2 \sqrt{5}|c|$ is the same as in [15, Lem. 10]. However, the proof reported therein does not apply in the presence of the boundary perturbation p. Indeed, following the lines of [15, Lem. 10], one gets an estimate similar to (18) but with the occurrence of the extra term $|\Delta \dot{p}(\tau)|$ in the term evaluating the contribution of $\Delta p$ and $\Delta r$. We refine here the stability analysis in order to obtain the claimed estimate (18) involving only $\Delta p$, and not $\Delta \dot{p}$.

Proof. Let $N \geq 0$ be such that $\lambda_{N+1}<-2 \sqrt{5}|c|$. We define $\eta=-\lambda_{N+1} / 2>\sqrt{5}|c| \geq 0$, which is such that $\lambda_{n} \leq \lambda_{N+1}=-2 \eta<0$ for all $n \geq N+1$. Note that, in this proof, we always consider integers $n \geq N+1$. Let $\kappa \in(0, \min (\eta, \sigma))$ be arbitrarily given and to be specified later. We introduce, for $t \geq 0, \Delta v_{n}(t)=\Delta x_{n}(t)-$ $\Delta x_{n}(t-h(t))$, yielding

$$
\Delta \dot{x}_{n}(t)=\lambda_{n} \Delta x_{n}(t)-c \Delta v_{n}(t)+\left(a_{n}+\lambda_{n} b_{n}\right) \Delta u(t)
$$

for all $t \geq 0$. We also consider the series

$$
\begin{array}{lr}
S_{x}(t)=\sum_{n \geq N+1}\left|\Delta x_{n}(t)\right|^{2}, \quad t \geq-h_{M} ; \\
S_{v}(t)=\sum_{n \geq N+1}\left|\Delta v_{n}(t)\right|^{2}, & t \geq 0
\end{array}
$$

which are finite because $S_{x}(t) \leq\|\Delta X(t)\|^{2}$ and $S_{v}(t) \leq$ $2 S_{x}(t)+2 S_{x}(t-h(t))$. Finally, we introduce for any $t_{1}<$ $t_{2}$ and any real-valued and continuous function $\psi$ the notation $\mathcal{I}\left(\psi, t_{1}, t_{2}\right)=\int_{t_{1}}^{t_{2}} e^{-2 \eta\left(t_{2}-\tau\right)}|\psi(\tau)| \mathrm{d} \tau$. We have $\mathcal{I}\left(\psi, t_{1}, t_{2}\right) \leq \frac{1-e^{-2(\eta-\kappa)\left(t_{2}-t_{1}\right)}}{2(\eta-\kappa)} \sup _{\tau \in\left[t_{1}, t_{2}\right]} e^{-2 \kappa\left(t_{2}-\tau\right)}|\psi(\tau)|$ and $\mathcal{I}\left(\psi, t_{1}, t_{2}\right)^{2} \leq \frac{1-e^{-2 \eta\left(t_{2}-t_{1}\right)}}{2 \eta} \mathcal{I}\left(\psi^{2}, t_{1}, t_{2}\right)$. By integrating (19), we obtain for $t \geq h_{M}$

$$
\Delta v_{n}(t)=\left\{e^{\lambda_{n} h(t)}-1\right\} \Delta x_{n}(t-h(t))
$$

$$
+\int_{t-h(t)}^{t} e^{\lambda_{n}(t-\tau)}\left\{-c \Delta v_{n}(\tau)+\left(a_{n}+\lambda_{n} b_{n}\right) \Delta u(\tau)\right\} \mathrm{d} \tau
$$

hence, using $\lambda_{n} \leq-2 \eta$,

$$
\begin{aligned}
& \left|\Delta v_{n}(t)\right| \leq\left|\Delta x_{n}(t-h(t))\right|+|c| \mathcal{I}\left(\Delta v_{n}, t-h(t), t\right) \\
& \quad+\left|a_{n}\right| \mathcal{I}(\Delta u, t-h(t), t) \\
& \quad+\left|b_{n}\right|\left|\lambda_{n} \int_{t-h(t)}^{t} e^{\lambda_{n}(t-\tau)} \Delta u(\tau) \mathrm{d} \tau\right| .
\end{aligned}
$$

Since $\kappa<\eta$ we have $\left|\lambda_{n} \int_{t-h(t)}^{t} e^{\lambda_{n}(t-\tau)} \Delta u(\tau) \mathrm{d} \tau\right| \leq$ $\frac{2 \eta}{2 \eta-\kappa} \sup _{\tau \in[t-h(t), t]} e^{-\kappa(t-\tau)}|\Delta u(\tau)|$ because $\lambda_{n} \leq$ $-2 \eta<-\eta<-\kappa<0$. Combining the two latter estimates and using Young's inequality we obtain

$$
\begin{aligned}
& \left|\Delta v_{n}(t)\right|^{2} \leq 4\left|\Delta x_{n}(t-h(t))\right|^{2}+\gamma_{1}|c|^{2} \mathcal{I}\left(\Delta v_{n}^{2}, t-h(t), t\right) \\
& \quad+\gamma_{1}\left|a_{n}\right|^{2} \mathcal{I}\left(\Delta u^{2}, t-h(t), t\right) \\
& \quad+\frac{16 \eta^{2}}{(2 \eta-\kappa)^{2}}\left|b_{n}\right|^{2} \sup _{\tau \in[t-h(t), t]} e^{-2 \kappa(t-\tau)}|\Delta u(\tau)|^{2}
\end{aligned}
$$

for all $t \geq h_{M}$ where $\gamma_{1}=\frac{2}{\eta}\left(1-e^{-2 \eta h_{M}}\right)$. Summing for $n \geq N+1$, we obtain for $t \geq h_{M}$

$$
\begin{aligned}
S_{v}(t) \leq & 4 S_{x}(t-h(t))+\gamma_{2}(\kappa)|c|^{2} \sup _{\tau \in[t-h(t), t]} e^{-2 \kappa(t-\tau)} S_{v}(\tau) \\
& +\gamma_{3}(\kappa) \sup _{\tau \in[t-h(t), t]} e^{-2 \kappa(t-\tau)}|\Delta u(\tau)|^{2}
\end{aligned}
$$

where $a=\mathcal{A}_{c} L \mathbb{1}, b=-L \mathbb{1}, \gamma_{2}(\kappa)=\frac{1}{\eta(\eta-\kappa)}(1-$ $\left.e^{-2 \eta h_{M}}\right)\left(1-e^{-2(\eta-\kappa) h_{M}}\right)$ and $\gamma_{3}(\kappa)=\gamma_{2}(\kappa)\|a\|^{2}+$ $\frac{16 \eta^{2}}{(2 \eta-\kappa)^{2}}\|b\|^{2}$. This implies that, for all $t \geq h_{M}$,

$$
\begin{aligned}
& \sup _{\tau \in\left[h_{M}, t\right]} e^{2 \kappa \tau} S_{v}(\tau) \leq 4 e^{2 \kappa h_{M}} \sup _{\tau \in\left[0, t-h_{m}\right]} e^{2 \kappa \tau} S_{x}(\tau) \quad(20) \\
& +\gamma_{2}(\kappa)|c|^{2} \sup _{\tau \in[0, t]} e^{2 \kappa \tau} S_{v}(\tau)+\gamma_{3}(\kappa) \sup _{\tau \in[0, t]} e^{2 \kappa \tau}|\Delta u(\tau)|^{2} .
\end{aligned}
$$

Integrating now (19) on $[0, t]$ for $t \geq 0$, using again $\lambda_{n} \leq$ $-2 \eta$, and proceeding as in the previous paragraph, we infer that, for all $t \geq 0$,

$$
\begin{aligned}
S_{x}(t) \leq & 4 e^{-2 \kappa t} S_{x}(0)+\gamma_{4}(\kappa)|c|^{2} \sup _{\tau \in[0, t]} e^{-2 \kappa(t-\tau)} S_{v}(\tau) \\
& +\gamma_{5}(\kappa) \sup _{\tau \in[0, t]} e^{-2 \kappa(t-\tau)}|\Delta u(\tau)|^{2}
\end{aligned}
$$

where $\gamma_{4}(\kappa)=\frac{1}{\eta(\eta-\kappa)}$ and $\gamma_{5}(\kappa)=\gamma_{4}(\kappa)\|a\|^{2}+$ $\frac{16 \eta^{2}}{(2 \eta-\kappa)^{2}}\|b\|^{2}$. Combining (20-21) and noting that $S_{x}(0) \leq\|\Delta \Phi(0)\|^{2}$, we obtain for $t \geq h_{M}$

$$
\sup _{\tau \in\left[h_{M}, t\right]} e^{2 \kappa \tau} S_{v}(\tau) \leq 16 e^{2 \kappa h_{M}}\|\Delta \Phi(0)\|^{2}
$$




$$
+\xi(\kappa) \sup _{\tau \in[0, t]} e^{2 \kappa \tau} S_{v}(\tau)+\gamma_{6}(\kappa) \sup _{\tau \in[0, t]} e^{2 \kappa \tau}|\Delta u(\tau)|^{2}
$$

with $\gamma_{6}(\kappa)=\gamma_{3}(\kappa)+4 e^{2 \kappa h_{M}} \gamma_{5}(\kappa)$ and

$$
\begin{aligned}
& \xi(\kappa)=\gamma_{2}(\kappa)|c|^{2}+4 e^{2 \kappa h_{M}} \gamma_{4}(\kappa)|c|^{2} \\
& =\frac{|c|^{2}}{\eta(\eta-\kappa)}\left\{4 e^{2 \kappa h_{M}}+\left(1-e^{-2 \eta h_{M}}\right)\left(1-e^{-2(\eta-\kappa) h_{M}}\right)\right\} .
\end{aligned}
$$

Recalling from the design constraint $\lambda_{N+1}<-2 \sqrt{5}|c|$ that $\eta>\sqrt{5}|c|$, we have $5|c|^{2} / \eta^{2}<1$. Hence, a continuity argument at $\kappa=0$ shows the existence of $\kappa \in$ $(0, \min (\eta, \sigma))$ such that $0 \leq \xi(\kappa)<1$. We fix such a $\kappa \in(0, \min (\eta, \sigma))$ for the rest of the proof. Since all the considered supremums are finite, we deduce from the latter estimate that, for all $t \geq h_{M}$,

$$
\begin{aligned}
& \sup _{\tau \in\left[h_{M}, t\right]} e^{2 \kappa \tau} S_{v}(\tau) \leq \frac{16 e^{2 \kappa h_{M}}}{1-\xi}\|\Delta \Phi(0)\|^{2} \\
& +\frac{\xi}{1-\xi} \sup _{\tau \in\left[0, h_{M}\right]} e^{2 \kappa \tau} S_{v}(\tau)+\frac{\gamma_{6}}{1-\xi} \sup _{\tau \in[0, t]} e^{2 \kappa \tau}|\Delta u(\tau)|^{2}
\end{aligned}
$$

where we dropped the dependency of $\gamma_{6}, \xi$ on the parameter $\kappa$ which is now fixed. To conclude the proof, we need to estimate the term $\sup _{\tau \in[0, t]} e^{2 \kappa \tau} S_{v}(\tau)$ for $t \in\left[0, h_{M}\right]$. From the definition of $S_{v}$ we have, for any $t \in\left[0, h_{M}\right]$, $\sup _{\tau \in[0, t]} e^{2 \kappa \tau} S_{v}(\tau) \leq 4 e^{2 \kappa h_{M}} \sup _{\tau \in\left[-h_{M}, t\right]} S_{x}(\tau)$. From (14b) and recalling that $n \geq N+1$ with $\lambda_{n} \leq-2 \eta<$ $-2 \sqrt{5}|c|$, we have $\lambda_{n}-c \leq \lambda_{n}+|c|<-(2 \sqrt{5}-1)|c| \leq 0$ hence

$$
\begin{aligned}
\left|\Delta x_{n}(t)\right| \leq & \left|\Delta x_{n}(0)\right|+|c| \sqrt{h_{M}} \sqrt{\int_{0}^{t}\left|\Delta x_{n}(\tau-h(\tau))\right|^{2} \mathrm{~d} \tau} \\
& +\left(\left|a_{n}\right| h_{M}+\left|b_{n}\right| e^{|c| h_{M}}\right) \sup _{\tau \in[0, t]}|\Delta u(\tau)|
\end{aligned}
$$

for all $t \in\left[0, h_{M}\right]$. Using Young's inequality and summing for $n \geq N+1$, we obtain

$$
\begin{aligned}
S_{x}(t) \leq & 3 S_{x}(0)+3|c|^{2} h_{M}^{2} \sup _{\tau \in\left[-h_{M}, t-h_{m}\right]} S_{x}(\tau) \\
& +6\left(\|a\|^{2} h_{M}^{2}+\|b\|^{2} e^{2|c| h_{M}}\right) \sup _{\tau \in[0, t]}|\Delta u(\tau)|^{2}
\end{aligned}
$$

for all $t \in\left[0, h_{M}\right]$. This implies, for all $t \in\left[0, h_{M}\right]$,

$$
\begin{aligned}
\sup _{\tau \in[0, t]} & S_{x}(\tau) \leq 3\left(1+|c|^{2} h_{M}^{2}\right) \sup _{\tau \in\left[-h_{M}, 0\right]}\|\Delta \Phi(\tau)\|^{2} \\
+ & +3|c|^{2} h_{M}^{2} \sup _{\tau \in\left[0, \max \left(t-h_{m}, 0\right)\right]} S_{x}(\tau) \\
& +6\left(\|a\|^{2} h_{M}^{2}+\|b\|^{2} e^{2|c| h_{M}}\right) \sup _{\tau \in[0, t]}|\Delta u(\tau)|^{2} .
\end{aligned}
$$

By a simple induction argument (since $h_{m}>0$ ), we obtain the existence of a constant $\gamma_{7}>0$ such that, for all $t \in\left[0, h_{M}\right], \sup _{\tau \in[0, t]} S_{x}(\tau) \leq \gamma_{7} \sup _{\tau \in\left[-h_{M}, 0\right]}\|\Delta \Phi(\tau)\|^{2}+$ $\gamma_{7} \sup _{\tau \in[0, t]}|\Delta u(\tau)|^{2}$. We deduce (see beginning of this paragraph) the existence of a constant $\gamma_{8}>0$ such that, for all $t \in\left[0, h_{M}\right], \sup _{\tau \in[0, t]} e^{2 \kappa \tau} S_{v}(\tau) \leq$ $\gamma_{8} \sup _{\tau \in\left[-h_{M}, 0\right]}\|\Delta \Phi(\tau)\|^{2}+\gamma_{8} \sup _{\tau \in[0, t]} e^{2 \kappa \tau}|\Delta u(\tau)|^{2}$. Combining this latter estimate with (22), we infer the existence of a constant $\gamma_{9}>0$ such that, for all $t \geq 0$,

$$
\begin{aligned}
\sup _{\tau \in[0, t]} e^{2 \kappa \tau} S_{v}(\tau) \leq & \gamma_{9} \sup _{\tau \in\left[-h_{M}, 0\right]}\|\Delta \Phi(\tau)\|^{2} \\
& +\gamma_{9} \sup _{\tau \in[0, t]} e^{2 \kappa \tau}|\Delta u(\tau)|^{2} .
\end{aligned}
$$

Substituting this estimate into (21), we obtain the existence of a constant $\gamma_{10}>0$ such that, for all $t \geq 0, S_{x}(t) \leq \gamma_{10} e^{-2 \kappa t} \sup _{\tau \in\left[-h_{M}, 0\right]}\|\Delta \Phi(\tau)\|^{2}+$ $\gamma_{10} \sup _{\tau \in[0, t]} e^{-2 \kappa(t-\tau)}|\Delta u(\tau)|^{2}$. The claimed estimate (18) now directly follows from the assumption that $u$ satisfies (17) and the fact that $0<\kappa<\sigma$.

\subsection{Completion of the proof of Theorem 1}

By applying first the result of Subsection 4.1 and then the result of Subsection 4.2, the claimed estimate (15) follows from $|\Delta \zeta(t)| \leq\left\|\Delta Y_{a}(t)\right\|,\|\Delta X(t)\| \leq$ $\left\|\Delta Y_{a}(t)\right\|+\sqrt{\sum_{n \geq N+1}\left|\Delta x_{n}(t)\right|^{2}}$, and (14c). This completes the proof of Theorem 1 .

\section{Setpoint regulation assessment}

We now address the setpoint regulation of the closedloop system for classical solutions.

Theorem 2 Under the assumptions of Theorem 1, and for the same constant $\kappa>0$, there exist constants $\bar{C}_{2}, \bar{C}_{3}>0$ such that, for all $h \in \mathcal{C}^{1}\left(\mathbb{R}_{+}\right)$with $h_{m} \leq h(t) \leq h_{M}$ and so that $t \mapsto t-h(t)$ crosses 0 a finite number of times, $\Phi \in \mathcal{C}^{1}\left(\left[-h_{M}, 0\right] ; \mathcal{H}\right)$ with $\Phi(0) \in D(\mathcal{A}), \zeta_{0} \in \mathcal{C}^{1}\left(\left[-h_{M}, 0\right]\right), p \in \mathcal{C}^{2}\left(\mathbb{R}_{+}\right)$, and $r \in \mathcal{C}^{1}\left(\mathbb{R}_{+}\right)$, all such that the compatibility condition (13) holds, we have, for all $t \geq 0$,

$$
\begin{aligned}
& |[X(t)](1)-r(t)| \leq \\
& \bar{C}_{2} e^{-\kappa t}\left\{\sup _{\tau \in\left[-h_{M}, 0\right]}\left(\|\Delta \Phi(\tau)\|+\left|\Delta \zeta_{0}(\tau)\right|\right)+\left\|\mathcal{A}_{c} \Delta \Phi(0)\right\|\right\} \\
& +\bar{C}_{3} \sup _{\tau \in[0, t]} e^{-\kappa(t-\tau)}(|\Delta p(\tau)|+|\Delta \dot{p}(\tau)|+|\Delta r(\tau)|)
\end{aligned}
$$

Corollary 3 In the context of Theorem 2, assume that $r(t) \rightarrow r_{e}, p(t) \rightarrow p_{e}$, and $\dot{p}(t) \rightarrow 0$ as $t \rightarrow+\infty$. Then $[X(t)](1) \rightarrow r_{e}$ as $t \rightarrow+\infty$ with exponential vanishing of the contribution of the initial conditions.

Proof of Theorem 2. Recalling that, for classical solutions, $W(t)=X(t)-L u(t) \in D\left(\mathcal{A}_{c, 0}\right)$, and since 
$W_{e}=X_{e}-L u_{e} \in D\left(\mathcal{A}_{c, 0}\right)$ with $X_{e}(1)=W_{e}(1)=r_{e}$, we have $|[X(t)](1)-r(t)| \leq\left|[W(t)](1)-r_{e}\right|+|\Delta r(t)| \leq$ $|[\Delta W(t)](1)|+|\Delta r(t)|$. To obtain (24), we only need to investigate the term $|[\Delta W(t)](1)|$. To do so, since $\lambda_{n} \sim-a n^{2} \pi^{2}$ as $n \rightarrow+\infty$, let $\delta>0$ and an integer $M \geq N$ be such that $\lambda_{n} \leq-(2 \kappa+\delta)<0$ and $\left|\lambda_{n}\right| \leq$ $\left|\lambda_{n}\right|^{\overline{2}}$ for all $n \geq M+1$. Then we have $|[\Delta W(t)](1)|=$ $\left|\sum_{n \geq 0} \Delta w_{n}(t) e_{n}(1)\right| \leq \sqrt{\sum_{n=0}^{M}\left|e_{n}(1)\right|^{2}}\|\Delta W(t)\|+$ $\sqrt{\sum_{n \geq M+1} \frac{\left|e_{n}(1)\right|^{2}}{\left|\lambda_{n}\right|}} \sqrt{\sum_{n \geq M+1}\left|\lambda_{n}\right|\left|\Delta w_{n}(t)\right|^{2}}$ where it can be seen from $\lambda_{n} \sim-a n^{2} \pi^{2}$ and $e_{n}(1)=O(1)$ as $n \rightarrow$ $+\infty$ that $\sum_{n>M+1} \frac{\left|e_{n}(1)\right|^{2}}{\left|\lambda_{n}\right|}<\infty$. From Theorem 1 and since $\|\Delta W(t)\| \leq\|\Delta X(t)\|+\|L\||\Delta u(t)|$, we only need to study the term $\sum_{n \geq M+1}\left|\lambda_{n}\right|\left|\Delta w_{n}(t)\right|^{2}$ to conclude that (24) holds. In the sequel we always consider integers $n \geq M+1$. From (14b) and recalling that $\Delta w_{n}(t)=$ $\Delta x_{n}(t)+b_{n} \Delta u(t)$, we have for $t \geq 0$ that $\Delta \dot{w}_{n}(t)=$ $\lambda_{n} \Delta w_{n}(t)-c \Delta v_{n}(t)+a_{n} \Delta u(t)+b_{n} \bar{\Delta} \dot{u}(t)$ with $\Delta v_{n}(t)=$ $\Delta x_{n}(t)-\Delta x_{n}(t-h(t))$. Then we obtain after integration on $[0, t]$ that $\sqrt{\left|\lambda_{n}\right|}\left|\Delta w_{n}(t)\right| \leq e^{\lambda_{n} t} \sqrt{\left|\lambda_{n}\right|}\left|\Delta w_{n}(0)\right|+$ $|c| \mathcal{J}_{1, n}(t)+\left|a_{n}\right| \mathcal{J}_{2, n}(t)+\left|b_{n}\right| \overline{\mathcal{J}}_{3, n}(t)$ for all $t \geq 0$ and $n \geq M+1$ with $\mathcal{J}_{1, n}(t)=\sqrt{\left|\lambda_{n}\right|} \int_{0}^{t} e^{\lambda_{n}(t-\tau)}\left|\Delta v_{n}(\tau)\right| \mathrm{d} \tau$, $\mathcal{J}_{2, n}(t)=\sqrt{\left|\lambda_{n}\right|} \int_{0}^{t} e^{\lambda_{n}(t-\tau)}|\Delta u(\tau)| \mathrm{d} \tau$, and $\mathcal{J}_{3, n}(t)=$ $\sqrt{\left|\lambda_{n}\right|} \int_{0}^{t} e^{\lambda_{n}(t-\tau)}|\Delta \dot{u}(\tau)| \mathrm{d} \tau$. Using $\lambda_{n} \leq-(2 \kappa+$ $\delta$ ) and $\left|\lambda_{n}\right| \leq\left|\lambda_{n}\right|^{2}$ for all $n \geq M+1$, we obtain that $\mathcal{J}_{1, n}(t) \leq \sqrt{\int_{0}^{t} e^{-(2 \kappa+\delta)(t-\tau)}\left|\Delta v_{n}(\tau)\right|^{2} \mathrm{~d} \tau}$, $\mathcal{J}_{2, n}(t) \leq 2 \sup _{\tau \in[0, t]} e^{-\kappa(t-\tau)}|\Delta u(\tau)|$, and $\mathcal{J}_{3, n}(t) \leq$ $2 \sup _{\tau \in[0, t]} e^{-\kappa(t-\tau)}|\Delta \dot{u}(\tau)|$. Combining the four latter inequalities, using next Young's inequality, and finally summing for $n \geq M+1$, we obtain that

$$
\begin{aligned}
& \sum_{n \geq M+1}\left|\lambda_{n}\right|\left|\Delta w_{n}(t)\right|^{2} \leq 4 e^{-2 \kappa t} \sum_{n \geq M+1}\left|\lambda_{n}\right|\left|\Delta w_{n}(0)\right|^{2} \\
& +4|c|^{2} \int_{0}^{t} e^{-(2 \kappa+\delta)(t-\tau)} \sum_{n \geq M+1}\left|\Delta v_{n}(\tau)\right|^{2} \mathrm{~d} \tau \\
& +16\|a\|^{2} \sup _{\tau \in[0, t]} e^{-2 \kappa(t-\tau)}|\Delta u(\tau)|^{2} \\
& +16\|b\|^{2} \sup _{\tau \in[0, t]} e^{-2 \kappa(t-\tau)}|\Delta \dot{u}(\tau)|^{2}
\end{aligned}
$$

for all $t \geq 0$. Since $M \geq N$, we have $\sum_{n \geq M+1}\left|\Delta v_{n}(\tau)\right|^{2} \leq$ $S_{v}(\tau)$. Hence, we obtain from (23) that

$$
\begin{aligned}
& \int_{0}^{t} e^{-(2 \kappa+\delta)(t-\tau)} \sum_{n \geq M+1}\left|\Delta v_{n}(\tau)\right|^{2} \mathrm{~d} \tau \leq \\
& \frac{\gamma_{9}}{\delta} e^{-2 \kappa t} \sup _{\tau \in\left[-h_{M}, 0\right]}\|\Delta \Phi(\tau)\|^{2}+\frac{\gamma_{9}}{\delta} \sup _{\tau \in[0, t]} e^{-2 \kappa(t-\tau)}|\Delta u(\tau)|^{2}
\end{aligned}
$$

The two latter inequalities imply the existence of a con- stant $\gamma_{11}>0$ such that

$$
\begin{aligned}
& \frac{1}{\gamma_{11}} \sum_{n \geq M+1}\left|\lambda_{n} \| \Delta w_{n}(t)\right|^{2} \leq \\
& e^{-2 \kappa t} \sum_{n \geq M+1}\left|\lambda_{n}\left\|\left.\Delta w_{n}(0)\right|^{2}+e^{-2 \kappa t} \sup _{\tau \in\left[-h_{M}, 0\right]}\right\| \Delta \Phi(\tau) \|^{2}\right. \\
& +\sup _{\tau \in[0, t]} e^{-2 \kappa(t-\tau)}|\Delta u(\tau)|^{2}+\sup _{\tau \in[0, t]} e^{-2 \kappa(t-\tau)}|\Delta \dot{u}(\tau)|^{2}
\end{aligned}
$$

for all $t \geq 0$. Since $\Delta W(0) \in D\left(\mathcal{A}_{c, 0}\right)$ and $\left|\lambda_{n}\right| \leq\left|\lambda_{n}\right|^{2}$ for all $n \geq M+1$, we note that $\sum_{n>M+1}\left|\lambda_{n}\right|\left|\Delta w_{n}(0)\right|^{2} \leq$ $\left\|\mathcal{A}_{c, 0} \Delta W(0)\right\|^{2} \leq 2\left\|\mathcal{A}_{c} \Delta X(0)\right\|^{2}+2\left\|\mathcal{A}_{c} L\right\|^{2}|\Delta u(0)|^{2}$ with $\Delta X(0)=\Delta \Phi(0)$ and $|\Delta u(0)| \leq\|K\|\left\|\Delta Y_{a}(0)\right\|+$ $|\Delta p(0)| \leq\|K\|\left(\|\Delta \Phi(0)\|+\left|\Delta \zeta_{0}(0)\right|\right)+|\Delta p(0)|$. To conclude the proof, it is sufficient to study the two last terms of (25). The estimation of the term involving $\Delta u$ immediately follows from (15). Hence, only the term involving $\Delta \dot{u}$ needs to be investigated. From (14a) and (14c), we have, for all $t \geq 0,|\Delta \dot{u}(t)| \leq\|K\|\left\|\Delta \dot{Y}_{a}(t)\right\|+|\Delta \dot{p}(t)|$ with $\left\|\Delta \dot{Y}_{a}(t)\right\| \leq\left\|A_{K}-c I\right\|\left\|\Delta Y_{a}(t)\right\|+|c| \| \Delta Y_{a}(t-$ $h(t))\|+\| B_{a} \||\Delta \bar{p}(t)|+|\Delta r(t)|$. The claimed conclusion now follows from $\left\|\Delta Y_{a}(\tau)\right\| \leq\|\Delta X(\tau)\|+|\Delta \zeta(\tau)|$ for $\tau \geq-h_{M}$ and (15).

Remark 6 In the context of Theorem 2 dealing with classical solutions, the stability result stated by Theorem 1 can be strengthen as follows. First, it can be shown similarly to [19, Eq. 42] that $\left\|f^{\prime}\right\|^{2}=$ $-\cot (\theta)|f(1)|^{2}+\frac{b+c}{a}\|f\|^{2}-\frac{1}{a} \sum_{n \geq 0} \lambda_{n}\left|\left\langle f, e_{n}\right\rangle\right|^{2}$ for any $f \in D\left(\mathcal{A}_{c, 0}\right)$. Considering classical solutions, we can apply this identity to $\Delta W(t) \in D\left(\mathcal{A}_{c, 0}\right)$ where we note that estimates of $\|\Delta W(t)\|$ and $|[\Delta W(t)](1)|$ are provided by Theorem 1 and Theorem 2, respectively, while the series $\sum_{n>0}\left|\lambda_{n}\right|\left|\Delta w_{n}(t)\right|^{2}$ has been evaluated in the proof of Theorem 2. Since $\Delta X(t)=\Delta W(t)+L \Delta u(t) \in D\left(\mathcal{A}_{c}\right) \subset H^{2}(0,1)$ with $\left\|[L \Delta u(t)]^{\prime}\right\|=\frac{2}{\sqrt{3}}|\Delta u(t)|$, we infer that $\left\|[\Delta X(t)]^{\prime}\right\|$, and hence $\|\Delta X(t)\|_{H^{1}(0,1)}$, is upper bounded by a term similar (i.e., with different constants $\bar{C}_{i}$ ) to the righthand side of (24). If we further make the assumptions of Corollary 3, we obtain that $X(t)$ converges in $H^{1}(0,1)$ norm and hence, by the continuous embedding $H^{1}(0,1) \subset \mathcal{C}^{0}([0,1])$, in $L^{\infty}$ norm to $X_{e}$ when $t \rightarrow+\infty$.

\section{Robustness with respect to delay mismatches}

In the previous sections, we have assumed the perfect knowledge of the state-delay $h$. This was used to build the dynamics of the integral component $\zeta$ given by (9). In this section, we discuss the robustness of the proposed control strategy with respect to delay mismatches. Assume that we dispose of an estimate $\hat{h}$ of the actual delay $h$ such that $|\hat{h}-h| \leq \delta$ for some constant $\delta>0$. In this case, we replace the integral component $\zeta$, originally 
defined by (9), by the following dynamics:

$$
\begin{aligned}
\dot{\zeta}(t)= & \sum_{n=0}^{N} x_{n}(t) e_{n}(1)+c\{\zeta(t-\hat{h}(t))-\zeta(t)\} \\
& +\alpha u(t)-r(t), \\
\zeta(\tau)= & \zeta_{0}(\tau), \quad \tau \in\left[-h_{M}, 0\right]
\end{aligned}
$$

Assuming that $\hat{h}$ satisfies the same assumptions as $h$, the only difference comparing to the previous developments occurs in the study of the truncated model. More precisely, the closed-loop truncated model, originally given by (14a) and (14d), becomes:

$$
\begin{aligned}
\Delta \dot{Y}_{a}(t)= & A_{K} \Delta Y_{a}(t)+c\left\{\Delta Y_{a}(t-h(t))-\Delta Y_{a}(t)\right\} \\
& +E_{c}\left\{\Delta Y_{a}(t-\hat{h}(t))-\Delta Y_{a}(t-h(t))\right\} \\
& +B_{a} \Delta p(t)+\Delta \Gamma(t) \\
\Delta Y_{a}(\tau)= & \Delta Y_{\Phi, a}(\tau), \quad \tau \in\left[-h_{M}, 0\right]
\end{aligned}
$$

with $E_{c}=\operatorname{diag}(0, \ldots, 0, c) \in \mathbb{R}^{(N+2) \times(N+2)}$. Provided a suitable choice of the feeback gain $K$, the existence of a maximal delay mismatch $\delta>0$ such that (27) is exponentially ISS with respect to the exogenous signals $\Delta p$ and $\Delta r$ follows from the following lemma.

Lemma 6 Let $N \geq 1,0<h_{m}<h_{M}, \mathbf{A}, \mathbf{E} \in \mathbb{R}^{N \times N}$ and $c \in \mathbb{R}$. Assume that $\mathbf{A}$ is Hurwitz with simple eigenvalues $\mu_{1}, \ldots, \mu_{N} \in \mathbb{C}$ such that $\operatorname{Re} \mu_{n}<-3|c|$ for all $1 \leq n \leq N$. Then there exist constants $\delta, \sigma, C_{6}, C_{7}>0$ such that, for any $x_{0} \in \mathcal{C}^{0}\left(\left[-h_{M}, 0\right] ; \mathbb{R}^{N}\right)$, any $h_{i} \in$ $\mathcal{C}^{0}\left(\mathbb{R}_{+}\right)$with $i \in\{1,2,3\}, h_{m} \leq h_{i}(t) \leq h_{M}$, and $\mid h_{2}-$ $h_{3} \mid \leq \delta$, and any $\tilde{q} \in \mathcal{C}^{0}\left(\mathbb{R}_{+} ; \mathbb{R}^{N}\right)$, the trajectory of

$$
\begin{aligned}
\dot{x}(t)= & \mathbf{A} x(t)+c\left\{x\left(t-h_{1}(t)\right)-x(t)\right\} \\
& +\mathbf{E}\left\{x\left(t-h_{2}(t)\right)-x\left(t-h_{3}(t)\right)\right\}+\tilde{q}(t), \\
x(\tau)= & x_{0}(\tau), \quad \tau \in\left[-h_{M}, 0\right]
\end{aligned}
$$

$$
\begin{aligned}
& \text { satisfies the estimate }\|x(t)\| \leq C_{6} e^{-\sigma t} \sup _{\tau \in\left[-h_{M}, 0\right]}\left\|x_{0}(\tau)\right\|+ \\
& C_{7} \sup _{\tau \in[0, t]} e^{-\sigma(t-\tau)}\|\tilde{q}(\tau)\| \text { for all } t \geq 0 .
\end{aligned}
$$

Hence, proceeding exactly as in the previous sections, we obtain the existence of a constant $\delta>0$ such that, when replacing the definition (9) of the integral component $\zeta$ by (26), the conclusions of Theorems 1 and 2 still hold true 2 for any estimated state-delay $\hat{h}$ satisfying the same assumptions as $h$ and with $|\hat{h}-h| \leq \delta$.

Proof. Introducing $v_{1}(t)=x(t)-x\left(t-h_{1}(t)\right), v_{2}(t)=$ $x\left(t-h_{2}(t)\right)-x\left(t-h_{3}(t)\right)$, and $q(t)=\mathbf{E} v_{2}(t)+\tilde{q}(t)$, we obtain from Lemma 4 that (16) holds. Since $\mathbf{A}$ is Hurwitz, we can assume that the constant $\sigma>0$ involved

\footnotetext{
${ }^{2}$ With constants $\bar{C}_{i}$ of the estimates (15) and (24) that are independent of a particularly selected $\hat{h}$.
}

in the latter equation (16) is further selected such that $\left\|e^{\mathbf{A} t}\right\| \leq M e^{-\sigma t}$ for all $t \geq 0$ and for some constant $M \geq 1$. Integrating (28) over either $\left[t-h_{3}(t), t-h_{2}(t)\right]$ if $h_{3}(t) \geq h_{2}(t)$ or $\left[t-h_{2}(t), t-h_{3}(t)\right]$ if $h_{2}(t) \geq h_{3}(t)$ for $t \geq h_{M}$, and combining these estimates, we obtain that

$$
\begin{aligned}
\sup _{\tau \in\left[h_{M}, t\right]} e^{\sigma \tau}\left\|v_{2}(\tau)\right\| \leq & \left\{e^{\delta\|\mathbf{A}\|}-1\right\} e^{\sigma h_{M}} \sup _{\tau \in[0, t]} e^{\sigma \tau}\|x(\tau)\| \\
& +\delta M|c| e^{\sigma h_{M}} \sup _{\tau \in[0, t]} e^{\sigma \tau}\left\|v_{1}(\tau)\right\| \\
& +\delta M\|\mathbf{E}\| e^{\sigma h_{M}} \sup _{\tau \in[0, t]} e^{\sigma \tau}\left\|v_{2}(\tau)\right\| \\
& +\delta M e^{\sigma h_{M}} \sup _{\tau \in[0, t]} e^{\sigma \tau}\|\tilde{q}(\tau)\|
\end{aligned}
$$

for all $t \geq h_{M}$. From (16), the identity $v_{1}(t)=$ $x(t)-x\left(t-h_{1}(t)\right)$, and based on a small gain argument, we can fix $\delta>0$ small enough (independently of $x_{0}, h_{i}$, and $\tilde{q}$ ) to obtain the existence of a constant $\gamma_{12}>0$ such that $\sup _{\tau \in\left[h_{M}, t\right]} e^{\sigma \tau}\left\|v_{2}(\tau)\right\| \leq$ $\gamma_{12} \sup _{\tau \in\left[-h_{M}, 0\right]}\left\|x_{0}(\tau)\right\|+\gamma_{12} \sup _{\tau \in\left[0, h_{M}\right]} e^{\sigma \tau}\left\|v_{2}(\tau)\right\|+$ $\gamma_{12} \sup _{\tau \in[0, t]} e^{\sigma \tau}\|\tilde{q}(\tau)\|$ for all $t \geq h_{M}$. Recalling that $v_{2}(t)=x\left(t-h_{2}(t)\right)-x\left(t-h_{3}(t)\right)$, one can estimate for $t \in\left[0, h_{M}\right]$ the term $\sup _{\tau \in[0, t]} e^{\sigma \tau}\left\|v_{2}(\tau)\right\|$ from (28) and the use of Grönwall's inequality. Combining with the latter estimate, we obtain the existence of a constant $\gamma_{13}>0$ such that $\sup _{\tau \in[0, t]} e^{\sigma \tau}\left\|v_{2}(\tau)\right\| \leq$ $\gamma_{13} \sup _{\tau \in\left[-h_{M}, 0\right]}\left\|x_{0}(\tau)\right\|+\gamma_{13} \sup _{\tau \in[0, t]} e^{\sigma \tau}\|\tilde{q}(\tau)\|$ for all $t \geq 0$. Since $q(t)=\mathbf{E} v_{2}(t)+\tilde{q}(t)$, the substitution of the latter estimate into (16) completes the proof.

\section{$7 \quad$ Simulation results}

We set $a=0.2, b=2, c=1$, and $\theta=\pi / 3$. The first eigenvalues of $\mathcal{A}_{c, 0}$ are approximately given by $\lambda_{0} \approx 2.301, \lambda_{1} \approx-1.668>-2 \sqrt{5}|c|$, and $\lambda_{2} \approx$ $-9.567<-2 \sqrt{5}|c|$. Hence we set $N=1$. The feedback gain $K \in \mathbb{R}^{1 \times 3}$ is computed such that $A_{K}=A_{a}+B_{a} K$ is Hurwitz with simple eigenvalues $\mu_{1}=-4, \mu_{2}=-5$, $\mu_{3}=-6$, selected so that $\mu_{n}<-3|c|$. The initial conditions of the plant and the integral component are set as $\phi(\tau, x)=10 \cos (3 \pi \tau) x(1-x)^{2}$ and $\zeta_{0}(\tau)=\cos (3 \pi \tau) \zeta_{a}$ where $\zeta_{a} \in \mathbb{R}$ is selected such that (13) holds. The numerical scheme consists of the modal approximation of the reaction-diffusion equation using its first 40 modes.

The behavior of the closed-loop system composed of (2), (9), and (12) is illustrated for the time varying delay $h(t)=1+\frac{1}{2} \sin (5 \pi t+\pi / 4)$ and the boundary perturbation $p(t)$ as shown in Fig. 1(d). The results are depicted in Fig. 1. During the 10 first seconds we observe that the control law achieves the stabilization of the closed-loop system: both the state and the regulated output converge to zero in spite of a constant perturbation $p(t)=1$. Then, in order to evaluate the setpoint tracking capabilities of the system ouput (see Thm. 2), the reference 


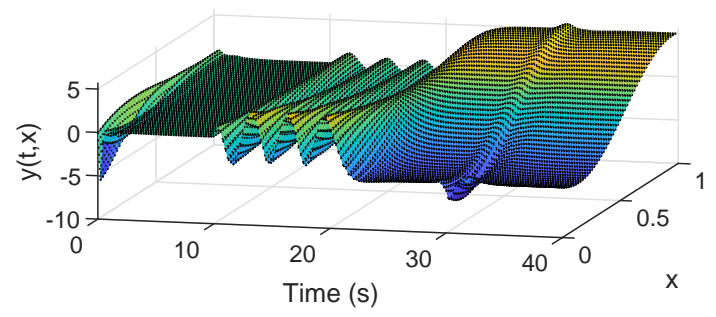

(a) State $y(t, x)$

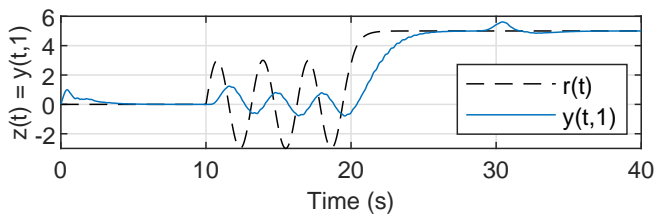

(b) Regulated output $z(t)=y(t, 1)$

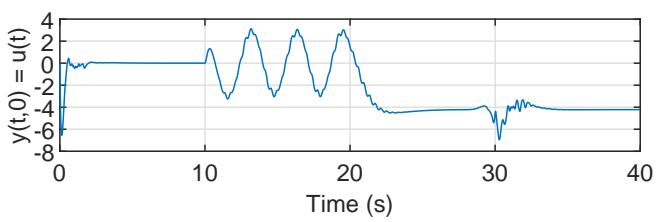

(c) Control input $y(t, 0)=u(t)$

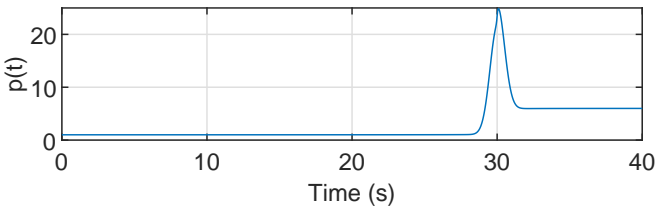

(d) Boundary perturbation $p(t)$

Fig. 1. Time evolution of the closed-loop system

signal is set as $r(t)=5$ for $t>20 \mathrm{~s}$ after an oscillatory transient. In conformity with the tracking estimate (24), we observe that the control strategy ensures the setpoint tracking of the reference signal $r(t)$ by the right Dirichlet trace $y(t, 1)$. Around $t=30 \mathrm{~s}$, the boundary perturbation $p(t)$ increases to reach (approximately) the value of 25 and then decreases to converge to the value of 6 . It is seen that the impact of this perturbation on both the state trajectory and the regulated output are successfully eliminated due to the presence of the integral component.

Finally, Fig. 2 illustrates the impact of delay mismatches on the closed-loop system performance. Here we set $\hat{h}=$ 1 while considering increasing values for the actual delay $h \in\{1,2,3,4\}$. The boundary perturbation is set as $p=1$. As expected, we observe a smooth degradation of the performances of the resulting closed-loop system.

\section{Conclusion}

This paper has investigated the boundary PI regulation control of a reaction-diffusion equation in the presence

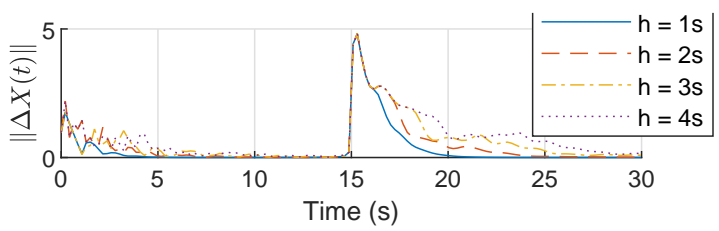

(a) Norm of the state $y(t, \cdot)$

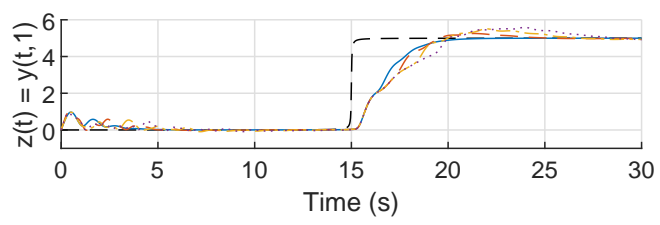

(b) Regulated output $z(t)=y(t, 1)$

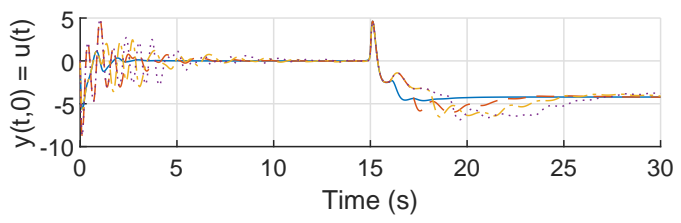

(c) Control input $y(t, 0)=u(t)$

Fig. 2. Impact of delay mismatches

of a state-delay in the reaction term. Our modal-based approach ensures the stability of the resulting closedloop system as well as the setpoint regulation of the right Dirichlet trace. Future research directions may be concerned with extensions to the PI regulation control of either linear wave equations or semilinear heat equations in the presence of a state-delay.

\section{References}

[1] Matthieu Barreau, Frédéric Gouaisbaut, and Alexandre Seuret. Practical stability analysis of a drilling pipe under friction with a PI-controller. IEEE Transactions on Control Systems Technology, 29(2):620-634, 2019.

[2] Georges Bastin, Jean-Michel Coron, and Simona Oana Tamasoiu. Stability of linear density-flow hyperbolic systems under PI boundary control. Automatica, 53:37-42, 2015.

[3] Jean-Michel Coron and Amaury Hayat. PI controllers for 1-D nonlinear transport equation. IEEE Transactions on Automatic Control, 64(11):4570-4582, 2019.

[4] Jean-Michel Coron and Emmanuel Trélat. Global steadystate controllability of one-dimensional semilinear heat equations. SIAM Journal on Control and Optimization, 43(2):549-569, 2004.

[5] Ruth Frances Curtain and Hans Zwart. An introduction to infinite-dimensional linear systems theory, volume 21 . Springer Science \& Business Media, 2012.

[6] Valrie Dos Santos, Georges Bastin, J-M Coron, and Brigitte d'Andréa Novel. Boundary control with integral action for hyperbolic systems of conservation laws: Stability and experiments. Automatica, 44(5):1310-1318, 2008.

[7] Tomoaki Hashimoto and Miroslav Krstic. Stabilization of reaction diffusion equations with state delay using boundary control input. IEEE Transactions on Automatic Control, 61(12):4041-4047, 2016. 
[8] Wen Kang and Emilia Fridman. Boundary control of delayed ODE-heat cascade under actuator saturation. Automatica, 83:252-261, 2017.

[9] Miroslav Krstic. Control of an unstable reaction-diffusion PDE with long input delay. Systems \& Control Letters, 58(10-11):773-782, 2009.

[10] Pierre-Olivier Lamare and Nikolaos Bekiaris-Liberis. Control of $2 \times 2$ linear hyperbolic systems: Backstepping-based trajectory generation and PI-based tracking. Systems 8 Control Letters, 86:24-33, 2015.

[11] Hugo Lhachemi and Christophe Prieur. Feedback stabilization of a class of diagonal infinite-dimensional systems with delay boundary control. IEEE Transactions on Automatic Control, in press, 66(1):105-120, 2020.

[12] Hugo Lhachemi, Christophe Prieur, and Robert Shorten. An LMI condition for the robustness of constant-delay linear predictor feedback with respect to uncertain time-varying input delays. Automatica, 109:108551, 2019.

[13] Hugo Lhachemi, Christophe Prieur, and Emmanuel Trélat. PI regulation of a reaction-diffusion equation with delayed boundary control. IEEE Transactions on Automatic Control, 66(4):1573-1587, 2020.

[14] Hugo Lhachemi, Christophe Prieur, and Emmanuel Trélat. PI regulation control of a 1-D semilinear wave equation. SIAM journal on control and optimization, 2021, in press.

[15] Hugo Lhachemi and Robert Shorten. Boundary feedback stabilization of a reaction-diffusion equation with Robin boundary conditions and state-delay. Automatica, 116:108931, 2020.

[16] Wim Michiels, Koen Engelborghs, Patrick Vansevenant, and Dirk Roose. Continuous pole placement for delay equations. Automatica, 38(5):747-761, 2002.

[17] Serge Nicaise and Cristina Pignotti. Stabilization of the wave equation with boundary or internal distributed delay. Differential and Integral Equations, 21(9-10):935-958, 2008.

[18] Seppo Pohjolainen. Robust multivariable PI-controller for infinite dimensional systems. IEEE Transactions on Automatic Control, 27(1):17-30, 1982.

[19] Christophe Prieur and Emmanuel Trélat. Feedback stabilization of a 1D linear reaction-diffusion equation with delay boundary control. IEEE Transactions on Automatic Control, 64(4):1415-1425, 2018.

[20] Alexandre Terrand-Jeanne, Vincent Andrieu, V Dos Santos Martins, and Chengzhong Xu. Adding integral action for open-loop exponentially stable semigroups and application to boundary control of PDE systems. IEEE Transactions on Automatic Control, 65(11):4481-4492, 2019.

[21] Alexandre Terrand-Jeanne, Vincent Andrieu, Melaz Tayakout-Fayolle, and Valerie Dos Santos Martins. Regulation of inhomogeneous drilling model with a PI controller. IEEE Transactions on Automatic Control, 65(1):58-71, 2020.

[22] Ngoc-Tu Trinh, Vincent Andrieu, and Cheng-Zhong Xu. Design of integral controllers for nonlinear systems governed by scalar hyperbolic partial differential equations. IEEE Transactions on Automatic Control, 62(9):4527-4536, 2017.

[23] Cheng-Zhong Xu and Hamadi Jerbi. A robust PI-controller for infinite-dimensional systems. International Journal of Control, 61(1):33-45, 1995.

[24] Cheng-Zhong Xu and Gauthier Sallet. Multivariable boundary PI control and regulation of a fluid flow system. Mathematical Control and Related Fields, 4(4):501-520, 2014. 